\title{
Ansiedad, depresión y estrés laboral asistencial en personal sanitario de un hospital de psiquiatría
}

\author{
Anxiety, depression and work-related stress in \\ healthcare personnel of a psychiatric hospital
}

\begin{abstract}
Gómez-Francisco, Laura Alin; Ocampo-Marín, Santiago; MontoyaNarváez, Diana Elizabeth; Valtierra-Contreras, Carmen Guadalupe; Rentería-Hernández, Fabián Leonel; Vázquez-Rincón, Martha Anahí; Reyna-García, Aurora Saraí; Rodríguez-Nava, Víctor Federico
\end{abstract}

\author{
Laura Alin Gómez-Francisco \\ Universidad Autónoma de Aguascalientes, México \\ Santiago Ocampo-Marín \\ Universidad Autónoma de Aguascalientes, México \\ Diana Elizabeth Montoya-Narváez \\ Universidad Autónoma de Aguascalientes, México \\ Carmen Guadalupe Valtierra-Contreras \\ Universidad Autónoma de Aguascalientes, México \\ Fabián Leonel Rentería-Hernández \\ Universidad Autónoma de Aguascalientes, México \\ Martha Anahí Vázquez-Rincón \\ Universidad Autónoma de Aguascalientes, México \\ Aurora Saraí Reyna-García \\ lic.aurorareyna18@gmail.com \\ Universidad Autónoma de Aguascalientes, México \\ Víctor Federico Rodríguez-Nava \\ Universidad Autónoma de Aguascalientes, México
}

\author{
Lux Médica \\ Universidad Autónoma de Aguascalientes, México \\ ISSN: 2007-1655 \\ Periodicidad: Cuatrimestral \\ vol. 16 , núm. 48,2021 \\ luxmedica.editorial@gmail.com \\ Recepción: 09 Noviembre 2020 \\ Aprobación: 24 Junio 2021 \\ URL: \\ https://revistas.uaa.mx/index.php/luxmedica/article/view/2918
}

Poítica de acceso abierto La Revista Lux Médica proporciona un acceso abierto a su contenido, basado en el principio de que ofrecer un acceso libre a las investigaciones ayuda a incrementar el intercambio global del conocimiento. La LM no cobra ni cobrará ningún cargo a sus lectores por concepto de suscripción, ni a los autores por enviar, procesar o publicar sus artículos. Como condición de publicación, los autores acuerdan liberar sus derechos de autor
Resumen: Introducción: El ambiente hospitalario de la salud mental requiere atención especial. Ante la prevalencia de cifras altas en torno al estrés laboral asistencial y sus principales consecuencias, como trastornos psicológicos (ansiedad y depresión), es necesario conocer el estado mental de las profesiones. Objetivo: Establecer la fuerza de relación entre la ansiedad, depresión y estrés laboral asistencial en personal sanitario de un hospital de psiquiatría. Materiales y métodos: Un estudio con diseño no experimental, relacional, transversal y prospectivo, evaluando estrés, ansiedad y depresión por medio del instrumento de Maslach Burnout Inventory, Ansiedad de Beck, Depresión de Hamilton; límites de espacio y tiempo en un hospital de psiquiatría de Aguascalientes durante el año 2020. Resultados: Se evaluó a 86 participantes y $2.3 \%$ presentó ansiedad severa, $5.8 \%$ mostró depresión mayor y el $3.5 \%$ sufrió estrés laboral asistencial alto. Se correlacionaron las variables: depresión y estrés laboral $(\mathrm{r}=0.22 \mathrm{p} \mathrm{p}=0.01)$, depresión y agotamiento emocional $(\mathrm{r}=0.569: \mathrm{p}=0.00)$, depresión y despersonalización $(r=0.407: p=0.00)$, depresión y realización personal $(\mathrm{p}=0.19)$, ansiedad y estrés laboral $(\mathrm{r}=0.351: \mathrm{p}=0.00)$, ansiedad y agotamiento emocional $(\mathrm{r}=0.483: \mathrm{p}=0.00)$, ansiedad $\mathrm{y}$ despersonalización $(\mathrm{r}=0.30: \mathrm{p}=0.00)$, ansiedad y realización personal ( $\mathrm{r}=-0.247: \mathrm{p}=0.01)$. Conclusión: El 36\% de trabajadores presentó estrés bajo y el $60.5 \%$ estrés moderado y áreas que desencadenan falta de contacto con la realidad, baja realización en tareas laborales, autoconcepto negativo y distanciamiento de actividades familiares/sociales. Existe una negación entorno a su propia salud mental, es necesario que se modifique el programa interno de atención hacia el personal sanitario.

Palabras clave: salud mental, ansiedad, depresión, estrés laboral, ambiente hospitalario.

Abstract: Introduction: The hospital environment of mental health requires special attention, given the high prevalence of work-related stress and its main consequences like psychological disorders (anxiety and depression), being necessary to know the mental state of professions. Objective: to establish the 
bajo una licencia compartida, específicamente la licencia de Creative Commons Reconocimiento-NoComercial-Compartir Igual 4.0 Internacional Esta licencia permite a cualquier persona compartir, copiar y redistribuir el material en cualquier medio o formato bajo los siguientes términos: - Dar crédito al autor del texto - No hace uso del material con propósitos comerciales - No transformar o modificar el material. Los autores ceden el derecho de la primera publicación a esta revista, pero conservarn sus derechos de autor, de tal forma que pueden realizar otros acuerdos contractuales independientes y adicionales para la distribución no exclusiva de la versión del artículo publicado en esta revista ( por ejemplo, incluirlo en un repositorio institucional o publicarlo en un libro) siempre que indiquen claramente que el trabajo se publicó por primera vez en ésta.

\section{(i) (2)}

Esta obra está bajo una Licencia Creative Commons Atribución NoComercial-CompartirIgual 4.0 Internacional.

Cómo citar este artículo: Gómez Francisco, L. A., Ocampo Marín, S., Montoya Narváez, D. E., Valtierra Contreras, C. G., Rentería Hernández, F. L., Vázquez Rincón, M. A., Reyna García, A. S., Rodríguez Nava, V. F. Ansiedad, depresión y estrés laboral asistencial en personal sanitario de un hospital de psiquiatría. Lux Médica, 16(48). Recuperado a partir de https://revistas.uaa.mx/index.php/lu xmedica/article/view/2918 strength of relationship between anxiety, depression, and workrelated stress in health personnel of a psychiatric hospital. Methods: a non-experimental, relational, cross-sectional, and prospective design study; evaluating stress, anxiety, and depression through the Maslach Burnout Inventory instrument, Beck's Anxiety, Hamilton's Depression, limits of space and time in a psychiatric hospital from Aguascalientes during 2020. Results: 86 participants were evaluated and $2.3 \%$ presented severe anxiety, $5.8 \%$ major depression and $3.5 \%$ high health care work stress; The variables were correlated: depression and work stress $(r=0.22: \mathrm{p}=$ $0.01)$, depression and emotional exhaustion $(r=0.569: p=0.00)$, depression and depersonalization $(r=0.407: p=0.00)$, depression and personal fulfillment $(\mathrm{p}=0.19)$, anxiety and work stress $(\mathrm{r}=$ $0.351: \mathrm{p}=0.00)$, anxiety and emotional exhaustion $(\mathrm{r}=0.483: \mathrm{p}=$ $0.00)$, anxiety and depersonalization $(r=0.30: p=0.00)$, anxiety and fulfillment personal $(r=-0.247: \mathrm{p}=0.01)$. Conclusion: $36 \%$ of workers presented low stress and $60.5 \%$ moderate stress and areas that trigger lack of contact with reality, low performance in work tasks, negative self-concept, and distancing from family / social activities. There is a denial around their mental health being necessary to modify the internal program of care for health personnel.

Keywords: mental health, anxiety, depression, work stress, hospital environment.

\section{INTRODUCCIÓN}

El síndrome de desgaste profesional fue introducido por Freudenberguer en los años setenta, y desarrollado por Maslach y Jackson como Burnout en la década de los ochenta. La literatura en castellano ofrece al menos diecinueve denominaciones de este fenómeno, entre ellos "estrés laboral asistencial" ${ }^{1,2}$ Este síndrome es una respuesta al estrés laboral crónico, conformado por actitudes y sentimientos negativos hacia las personas con las que se trabaja, y hacia el propio rol profesional, compuesto por tres dimensiones. ${ }^{3,4}$ En el ambiente hospitalario de la salud mental se requiere una especial atención, debido, principalmente, a que existen diferentes factores desencadenantes que limitan el desarrollo personal y laboral; entre ellos, ambiente físico de trabajo y contenidos del puesto, estrés por desempeño de roles, relaciones interpersonales, desarrollo de la carrera, estresores relacionados con aspectos organizacionales y fuentes extra organizacionales de estrés laboral, como relaciones trabajo-familia, considerándose a esta última, más una variable facilitadora que desencadenante.,

Dichos factores actúan directamente sobre el individuo y el grupo de trabajo. Entre las principales consecuencias del estrés laboral asistencial se encuentran: síntomas físicos, trastornos psicológicos (ansiedad, depresión), problemas conductuales, falta de atención en los procesos de cuidado e incremento de iatrogenias. ${ }^{7}$ En el ámbito de la salud mental no solamente los médicos están expuestos a estrés de origen asistencial, otras profesiones vinculadas a la asistencia también; es muy frecuente en el personal sanitario, como psicólogos, psiquiatras, enfermeras, terapeutas ocupacionales-familiares, consejeros, entre otros. ${ }^{3}$ 
Entre las alteraciones psiquiátricas más estudiadas como consecuencia del estrés laboral en personal sanitario, se ha resaltado el desarrollo de trastornos depresivos y de ansiedad. María Ovalles Pérez ${ }^{8}$ menciona en su estudio sobre estrés laboral, ansiedad y depresión en un hospital público que la prevalencia de estrés laboral es del $100 \%$ en el total de la muestra, la ansiedad del $63.07 \%$ y la depresión del $58.46 \%{ }^{8}$

Por otro lado, esto ha sido descrito en instituciones de psiquiatría por medio de las diferentes sub-escalas que conforman el MBI (Maslach Burnout Inventory). Ihosvany Basset Machado et al (2011) refiere resultados obtenidos en el personal de enfermería: una prevalencia de $85 \%$ con bajo nivel de agotamiento emocional, el $56.7 \%$ con bajo nivel de despersonalización y el $76.7 \%$ con un nivel bajo en la última escala con referencia a la realización personal, predominando un puntaje en el nivel bajo.?

En investigaciones donde se unifica el diagnóstico para el estrés laboral con el instrumento MBI, por la naturaleza de las dimensiones, las dos primeras directas y la última inversa, existen diferentes formas de obtener el diagnóstico y prevalencia, acorde al tipo de instrumento. Bajo este contexto, Walter Lizandro Arias Gallegos, ${ }^{10}$ en su estudio sobre personal de enfermería, refiere cifras del síndrome con $4.3 \%$ para leve, $91.5 \%$ para moderado y $4.3 \%$ para severo. ${ }^{10}$

Edgar Landa Ramírez et al. ${ }^{11}$ menciona en su estudio correlaciones entre la ansiedad, la depresión y las tres dimensiones, siendo significativas, positivas, con fuerzas de relación entre débiles y moderadas: depresión y burnout $(r=0.58 ; \mathrm{p}$ $<0.01)$, depresión y agotamiento emocional $(\mathrm{r}=0.55$; $\mathrm{p}<0.01)$, depresión $\mathrm{y}$ despersonalización $(\mathrm{r}=0.33 ; \mathrm{p}<0.01)$, depresión y realización personal $(\mathrm{r}=$ $0.33 ; \mathrm{p}<0.01)$, ansiedad y burnout $(\mathrm{r}=0.51 ; \mathrm{p}<0.01)$, ansiedad y agotamiento $(\mathrm{r}=0.45 ; \mathrm{p}<0.01)$, ansiedad y realización personal $(\mathrm{r}=0.34 ; \mathrm{p}<0.01)$; por último, ansiedad y despersonalización $(\mathrm{p}>0.05)$ no tienen relación. ${ }^{11}$

Partiendo de la información presentada en torno al estrés laboral asistencial, el objetivo general fue establecer la fuerza de relación entre la ansiedad, la depresión y el estrés laboral asistencial en personal sanitario de un hospital de psiquiatría.

\section{MATERIALES Y MÉTODOS}

Se trata de una investigación no experimental, cuantitativa, relacional, transversal y prospectiva; con personal sanitario de un hospital de psiquiatría en el estado de Aguascalientes, Ags, en el año 2020. Los criterios de selección fueron: ser personal sanitario, sin distinción de sexo o turno laboral, no consumir algún tipo de psicofármaco y que su cuestionario esté contestado en su totalidad. La técnica de selección se efectuó con una muestra final no probabilística por conveniencia, conformada de un total de 96 mediciones de los cuales cinco fueron de exclusión y cinco de eliminación, dejando un total de 86 participantes.

Se utilizó el Inventario de Ansiedad de Beck (BAI) elaborado por Beck y Steer en 1993, el cual incluye 21 ítems, posee un Alfa de Cronbach de 0.94, y se valora en una escala de 0-3. Las puntuaciones del BAI definen diferentes niveles de sintomatología ansiosa: 0-7 indica ansiedad mínima, 8-15 ansiedad leve, 16-25 ansiedad moderada y 26-63 ansiedad severa. ${ }^{12,13}$ 
Asimismo, se utilizó la Escala de Evaluación para la Depresión de Hamilton, versión y adaptación de Ramos-Brieva y Cordero14, reducida de 17 ítems. Esta posee un Alfa de Cronbach 0.92, se valora en una escala de 0-4 y establece la puntuación de 0-7 Sin Depresión, 8-12 Depresión menor, 13-17 Menos que Depresión mayor, 18-29 Depresión mayor y 30-52 Más que Depresión mayor. ${ }^{14}$

El instrumento de medida utilizado para el estrés laboral asistencial fue el Maslach Burnout Inventory, creado por Maslach y Jackson en 1981. Este cuestionario mide la frecuencia e intensidad de los ítems. El Alfa de Cronbach en las subescalas es: 0.89 para agotamiento emocional (AE), un 0.77 para despersonalización (DP), y 0.74 para realización personal (RP). ${ }^{15}$ Consta de 22 ítems en total y en él se pueden diferenciar tres subescalas, que miden, a su vez, las dimensiones que conforman el síndrome. ${ }^{16}$ Con siete niveles -0 a 6-, se utiliza un sistema de puntuación tipo Likert, $\mathrm{B} a j o(\mathrm{AE}=\mathrm{o}\langle 18$; $\mathrm{DP}=\mathrm{o}\langle 5 ; \mathrm{RP}=\mathrm{o}\rangle$ 40); Medio (AE 19-26; DP 6-9; RP 34 - 39); Alto (AE = o > 27; $\mathrm{DP}=\mathrm{o}>10$; $\mathrm{RP}=<33)$. La relación entre puntuación y grado de burnout es dimensional. Las puntuaciones de cada subescala se computan por separado y no se combinan ni se obtiene una puntuación total del MBI. En caso de obtener un bajo AE, una baja DP y una alta RP por cada dimensión, el riesgo es leve o bajo. En el resto de los casos hablaríamos de existencia de burnout alto o grave si se obtuviera un alto $\mathrm{AE}$, una alta DP y una baja RP; y moderado en el resto de las situaciones. ${ }^{17,18} \mathrm{~A}$ fin de proporcionar fiabilidad interna a esta muestra, se obtuvo un Alfa de Cronbach de los instrumentos y las tres dimensiones: BAI 0.92, EDH 0.83, AE 0.90, DP 0.67 y RP 0.88 .

Se registro ante la UAA y se aprobó con el código AEI-04-20. Además, se presentó el protocolo ante el hospital de psiquiatría y el ISSEA (Instituto de Servicios de Salud del Estado de Aguascalientes) para ser evaluado y contar con el registro, código 04 ISSEA-020/04. Previo a la aplicación de los instrumentos, contando con la autorización por parte del Comité Interno, una semana antes se acudió al Hospital de Psiquiatría a la reunión semanal de "secciones" para dar a conocer los investigadores y el estudio al personal sanitario. Así mismo, se le proporcionó información a cada participante, enfatizando en los siguientes aspectos: cordial saludo y presentación corta, una explicación clara y breve sobre la finalidad de la investigación, solicitud de su consentimiento verbal y por escrito en la hoja de consentimiento informado (el cual contiene aspectos sobre la confidencialidad, contacto y uso de la información recabada, apegado a lo dispuesto por la Ley General de Salud en materia de investigación), ${ }^{19}$ entrega de los cuestionarios, explicación de cómo estaba conformada la batería y el tiempo de llenado. Al terminar de contestarlo, se revisó que estuviera completo; de lo contrario, se solicitaba que se contestara en su totalidad. Finalmente, se agradeció la participación.

Una vez obtenidos todos los cuestionarios se unificaron e introdujeron a la matriz de datos, la cual fue analizada a través del software estadístico SPSS versión 25. Previo al análisis inferencial, se realizó la prueba de KolmogorovSmirnov para determinar la distribución de los datos, los cuales no presentaron distribución normal $(\mathrm{p}<0.05)$, de modo que se decidió realizar estadística no paramétrica. Se efectuaron medidas de resumen (tendencia central y posición) para las variables, y análisis de correlación con la prueba Rho de Spearman, se 
consideró un nivel de significancia de 0.05 . La obtención del diagnóstico y nivel del estrés laboral asistencial fue por medio del software Excel, con la función formato condicional para resaltar la información. Como hallazgo adicional, se obtuvieron análisis de regresión lineal simple y un modelo de fuerza de asociación con técnica no supervisada a través del software estadístico SPSS Modeler versión 18 , en el cual solo se consideraron variables significativas $(\mathrm{p}<0.05)$ por correlación de tipo marca.

\section{RESULTADOS}

\section{Variables sociodemográficas}

Se tuvieron 86 participantes en la muestra, de los cuales el 64\% ( $\mathrm{n}=55)$ corresponde al sexo femenino y $36 \%(\mathrm{n}=31)$ al masculino. Las mujeres representan más de la mitad del personal. Los datos arrojan que la edad mínima es de 21 años y la máxima de 65, y la mayoría tiene más de 30 años; es decir, el 64\% del personal sanitario está en una edad productiva laboral comprendida entre los 30 y 49 años. El estado civil sobresaliente es casado con 53.5\% ( $n=46)$, seguido de soltero, con $27.9 \%(n=24)$; divorciado, con $11.6 \%(n=10)$, y otros $7 \%(n=5)$; con ningún hijo como mínimo y un máximo de cuatro hijos, resultando la moda en 2 hijos.

Con el fin de proporcionar mayor información sobre las profesiones que están expuestas al estrés de tipo asistencial, se incluyeron en la investigación todas los que conforman a la institución además de la medicina. Predominó la profesión de enfermería (47.7\%), seguida por la Psicología (17.4\%), siendo Odontología la profesión con menor número de personal (2.3\%). El turno laboral con mayor personal sanitario es el matutino (44.2\%) y aquel con menos es la jornada acumulada, con el $12.8 \%$, ya que es el personal que labora en fines de semana y días festivos.

La antigüedad profesional de los participantes oscila entre una mínima de un año y una máxima de 42 , con una media recortada al $5 \%$ de 14.74 años, además de la antigüedad laboral en la institución media recortada al 5\% de 10.9 años, con un rango de 28 años y una moda de 10 años. El nivel ocupacional predominante es general con $72.1 \%$, de modo que la contratación es base con el $76.7 \%$; contrato, con el $18.6 \%$, y suplente, con el $4.7 \%$. El salario (en pesos mexicanos) de los participantes es de un mínimo de $\$ 3,500$ y un máximo de $\$ 45,000$, con una media recortada al 5\% de $\$ 15,709.30$ y una moda de $\$ 10,000$.

Además, se realizaron preguntas de interés en el cuestionario de datos sociodemográficos: el $88.4 \%$ niega tener una deuda superior a sus ingresos económicos; el 34.9\% afirma tener otro trabajo aparte del hospital; el 18.65\% padece de alguna enfermedad y tan solo el $17.4 \%$ lleva un tratamiento; el $61.6 \%$ realiza actividad física e, igualmente, un $61.6 \%$ practica un pasatiempo; el $82.6 \%$ niega acudir a terapia psicológica, siendo una prestación con la que cuenta el personal por parte de la institución, como periodo de desintoxicación; el 98.8\% no tiene algún diagnóstico de trastorno psicológico; el 10.5\% afirma tener un conflicto con un compañero en la institución, y el $46.5 \%$ de la muestra afirma tener algún conocimiento previo respecto a los instrumentos aplicados. 
La proporción de ansiedad evaluada refiere el $83.7 \%$ con ansiedad mínima, el $8.1 \%$ con ansiedad leve, el $5.8 \%$ con ansiedad moderada y el $2.3 \%$ con ansiedad severa entre el personal sanitario en general. De ellos, catalogándolos por profesión, la ansiedad mínima predomina en psiquiatría y odontología con un $100 \%$, siendo minorías los niveles altos; mientras que en enfermería el $4.9 \%$ tiene ansiedad severa, el $2.4 \%$ moderada y el $9.8 \%$ leve; en medicina el $10 \%$ tiene ansiedad moderada; en trabajo social el $12.5 \%$ tiene ansiedad moderada y otro $12.5 \%$ ansiedad leve; en nutrición el $33.3 \%$ tiene ansiedad moderada; en psicología el $6.7 \%$ tiene ansiedad moderada y otro $6.7 \%$ tiene ansiedad leve, y entre los terapistas el $25 \%$ presenta ansiedad leve.

\section{Escala para la depresión de Hamilton}

Entre el personal sanitario en general, la proporción sin datos de depresión evaluada fue del $83.7 \%$, mientras que el $10.5 \%$ presentó un nivel de depresión menor y el 5.8\% menos que depresión mayor.

La ausencia de depresión está presente en todas las profesiones, como medicina, psiquiatría y odontología con un $100 \%$. Así mismo, en enfermería el $7.3 \%$ tiene menos que depresión mayor y el $12.2 \%$ depresión menor; en trabajo social el 25\% tiene depresión menor; en nutrición el 33.3\% tiene menos que depresión mayor, y entre los terapistas el $25 \%$ tiene menos que depresión mayor.

\section{Agotamiento emocional}

La proporción del personal con un nivel bajo de agotamiento emocional fue del $86 \%$, con un $7 \%$ en el nivel medio e, igualmente, un $7 \%$ en nivel alto. Catalogándolos por profesión, todos refieren nivel bajo, como trabajo social, psiquiatría y odontología con un $100 \%$. En enfermería el $4.9 \%$ tiene un nivel alto y el $7.3 \%$ un nivel medio de agotamiento emocional; en medicina el $10 \%$ tiene un nivel alto; en nutrición el 33.3\% tiene un nivel alto; en psicología el 13.3\% tiene un nivel alto y, de igual forma, el $13.3 \%$ tiene nivel medio, y entre los terapistas el $25 \%$ tiene un nivel medio de agotamiento emocional. El 9.7\% de los hombres tiene un nivel medio (3.2\%) y un nivel alto (6.5\%), mientras que el $16.4 \%$ de las mujeres tiene un nivel medio $(9.1 \%)$ y alto $(7.3 \%)$ de agotamiento emocional.

\section{Despersonalización}

La proporción del personal sanitario con un nivel bajo de despersonalización es del $83.7 \%$, con un nivel medio de $10.5 \%$ y con un nivel alto de $5.8 \%$ de la muestra en general.

El nivel bajo está presente en todas las profesiones como psicología, psiquiatría, terapista y odontología con un 100\%, mientras que en enfermería el $7.3 \%$ tiene un nivel alto de despersonalización y el $14.6 \%$ tiene un nivel medio; en medicina el $10 \%$ tiene un nivel alto y un $20 \%$ un nivel medio; en trabajo social el $12.5 \%$ tiene un nivel medio, y en nutrición el $33.3 \%$ tiene un nivel alto de despersonalización. 
El $16.1 \%$ de los hombres tienen un nivel medio de despersonalización, y el $16.4 \%$ de las mujeres tiene un nivel medio (7.3\%) y alto (9.1\%). Los niveles de despersonalización altos en cuestión de turno laboral son de un $5.3 \%$ en el turno matutino, $11.1 \%$ en el vespertino y $9.1 \%$ en la jornada acumulada.

\section{Realización personal}

En una relación inversa, ya que a mayor puntuación es mayor la realización, los niveles de realización personal demuestran una alta proporción, del $45.3 \%$ en el nivel bajo, mientras que el $18.6 \%$ presenta un nivel medio y el $36 \%$ un nivel alto. Todas las profesiones poseen un nivel alto, aunque únicamente odontología se encuentra en un $100 \%$. Del resto de las profesiones, en enfermería el $24.4 \%$ tiene un nivel medio y el 39\% un nivel bajo de realización personal; en medicina el 10\% tiene un nivel medio y el 60\% tiene un nivel bajo; en trabajo social el 37.5\% tiene un nivel medio y otro $37.5 \%$ tiene un nivel bajo; en nutrición el $33.3 \%$ tiene un nivel bajo; en psicología el $13.3 \%$ tiene un nivel medio y el $66.7 \%$ presenta un nivel bajo; en psiquiatría el $66.7 \%$ tiene un nivel bajo, y entre los terapistas el 25\% tiene un nivel bajo de realización personal. El 51.6\% de los hombres tiene baja realización personal y el $12.9 \%$ nivel medio; y de las mujeres el $41.8 \%$ tienen un nivel bajo y el $21.8 \%$ medio.

\section{Estrés laboral asistencial}

El nivel de estrés laboral asistencial en el total de la muestra refiere un 36\% en nivel bajo, $60.5 \%$ en nivel moderado y un $3.5 \%$ en nivel alto. Los niveles predominantes son bajo y moderado en cada una de las profesiones y el nivel alto solo esta presenta en enfermería, medicina y nutrición, (Figura 1) eso corresponde a un estrés laboral crónico en el cual presentan una crisis de autoeficacia, afección cognitivo-aptitudinal en su desempeño laboral y personal.

Del total de los hombres, el 38.7\% presenta nivel bajo y el 61\% presenta nivel moderado del estrés asistencial; y, del total de las mujeres, el 34.5\% presenta nivel bajo, el 60\% nivel moderado y el 5.5\% nivel alto.

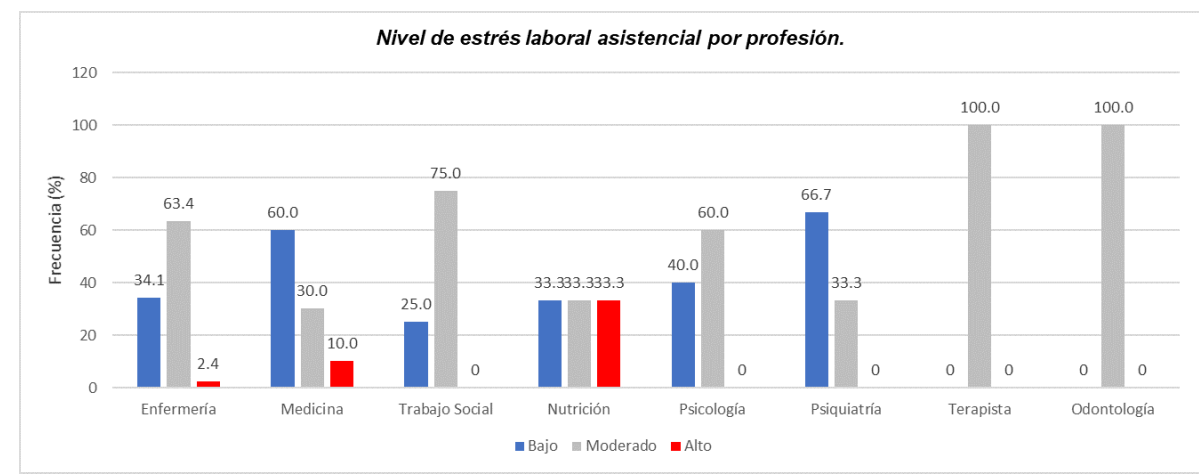

Figura 1. Nivel de estrés laboral asistencial por profesión

Fuente: Matriz de datos. Elaboración propia. Personal sanitario. Enfermería ( $\mathrm{n}=41)$, Medicina $(\mathrm{n}=10)$, Trabajo Social ( $n=8)$, Nutrición ( $n=3)$, Psicología ( $n=15)$, Psiquiatría ( $n=3)$, Terapista $(n=4)$, Odontología $(n=2)$. 


\section{Correlaciones}

Para determinar la fuerza de correlación y dirección de las variables se utilizó el valor del coeficiente de correlación de la prueba Rho de Spearman (Tabla 1) para dar el calificativo del grado (fuerza) de relación de cada una, el cual se muestran a continuación:

Tabla 1. Coeficiente de correlación de Spearman

\begin{tabular}{lllllll}
\hline & & AE & DP & RP & ELA & BAI \\
\hline \multirow{2}{*}{ BAI } & P valor & 0.000001 & 0.002193 & 0.010867 & 0.000463 & - \\
& Rho & $0.483^{* *}$ & $0.304^{* *}$ & $-0.247^{*}$ & $0.351^{* *}$ & - \\
\multirow{2}{*}{ EDH } & P valor & $5.4136 \mathrm{E}-9$ & 0.000051 & 0.194591 & 0.016823 & $4.7619 \mathrm{E}-7$ \\
& Rho & $0.569^{* *}$ & $0.407^{* *}$ & -0.094 & $0.229^{*}$ & $0.500^{* *}$ \\
\hline
\end{tabular}

Fuente: Matriz de datos, elaboración propia. Inventario ansiedad de Beck (BAI), Escala de Depresión de Hamilton (EDH), Dimensiones: Agotamiento Emocional (AE), Despersonalización (DP), Realización Personal (RP) y Estrés Laboral Asistencial (ELA). ( $\mathrm{n}=86)$. La correlación es significativa en el nivel $0,05^{*}-0,01^{* *}$ (unilateral).

La fuerza de relación entre la variable ansiedad y la dimensión agotamiento emocional es moderada $(r=0.48)$ y directa $(+)$, es decir a moderados niveles de agotamiento emocional es moderado el nivel de ansiedad presente en esta muestra. La fuerza de relación entre la variable ansiedad y la dimensión de despersonalización es baja $(\mathrm{r}=0.30)$ y directa $(+)$; por lo tanto, a bajos niveles de despersonalización, bajo es el nivel de ansiedad en la muestra. La fuerza de relación entre la variable ansiedad y la dimensión realización personal es baja $(\mathrm{r}=$ -0.24) e inversa (-); puesto que, a menores niveles de realización personal es mayor lo niveles de ansiedad. La fuerza de relación entre la variable ansiedad y la variable de estrés laboral asistencial es baja $(\mathrm{r}=0.35)$ y directa $(-)$; a medida que sea bajo el nivel de estrés laboral es bajo el nivel de ansiedad. la fuerza de relación entre la variable depresión y la dimensión agotamiento emocional es moderada ( $\mathrm{r}=$ $0.56)$ y directa (+); con un nivel moderado de agotamiento emocional también es moderado el nivel de depresión. La fuerza de relación entre la variable depresión y la dimensión despersonalización es moderada $(\mathrm{r}=0.40)$ y directa $(+)$; igualmente a niveles moderados de despersonalización es moderado el nivel de depresión. La fuerza de relación entre la variable depresión y la variable de estrés laboral asistencial es baja $(r=0.22)$ y directa $(+)$; a bajo estrés laboral es bajo el nivel de depresión. La fuerza de relación entra la variable ansiedad y depresión es moderada $(r=0.50)$ y directa $(+)$. No hay relación en esta muestra, entre la variable realización personal y depresión.

\section{Análisis de regresión lineal simple}

\section{Ansiedad}

El análisis de regresión lineal realizado para la variable síndrome de burnout como variable independiente y la variable ansiedad de Beck como variable dependiente confirma la existencia de una relación causal. 
El p valor para la constante (0.000) y el coeficiente síndrome de burnout (0.004) son significativos para poder establecer el modelo obteniendo la ecuación:

Nivel de ansiedad $=\mathbf{1 . 0 1 0}+\mathbf{0 . 3 8 1} \cdot$ nivel de síndrome de burnout

\section{Depresión}

En tanto que, al realizar el mismo procedimiento solo que con la variable depresión de Hamilton como dependiente e igual la variable síndrome de burnout como variable independiente confirma la existencia de una relación causal. El p valor para la constante $(0.000)$ y el coeficiente síndrome de burnout (0.002) son significativos para poder establecer el modelo obteniendo la ecuación:

Nivel de depresión $=0.999+0.329 \cdot$ nivel de síndrome de burnout

\section{Modelo}

Se generó un modelo de asociación con variables significativas, en el cual todas son consideradas de la misma jerarquía para poder establecer fuerzas de relación existentes entre dos o más variables simbólicas. Cada variable describe una característica presente en el total de la muestra extraídas de los ítems que componen los tres instrumentos aplicados (Figura 2).

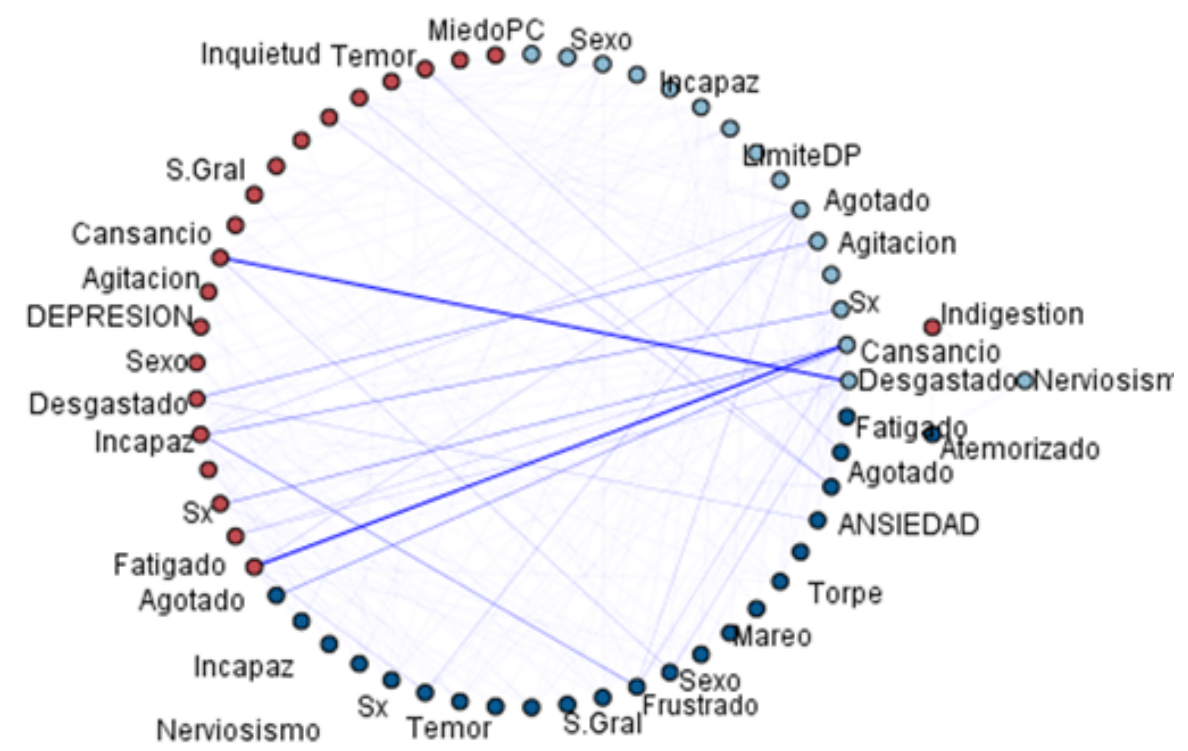

Figura 2. Gráfico de malla. Fuerza de asociación entre variables

Nota: las conexiones fuertes se indican con una línea gruesa, las conexiones medias se muestran con una línea de grosor normal, y las conexiones débiles se muestran con una línea puntead 


\section{Reglas de asociación}

Con respecto a la gráfica de malla, se formularon reglas de asociación que predicen con porcentaje de probabilidad la aparición de una consecuencia en base a uno a más antecedentes. A su vez, estas resultaron de una variedad de combinaciones posibles; por ello, solo se describen las más sobresalientes referente a la gráfica de malla.

- Sentirse desgastado en el trabajo existe una $100 \%$ de certeza de sentirse cansado

- Levantarse fatigado por las mañanas existe una $100 \%$ de certeza a sentirse cansado por trabajar todo el día con la gente

- Sentirse frustrado en el trabajo existe un $100 \%$ de certeza de ser incapaz de relajarse

- Sentirse inestable, levantarse fatigado por las mañanas y tener hijos tiene un $100 \%$ de certeza de sentirse cansado por trabajar todo el día con la gente

- Incapaz de relajarse, sentirse cansado por trabajar con la gente, levantarse fatigado por la mañana y sentirse emocionalmente agotado por el trabajo tiene una certeza del $100 \%$ de sentirse desgastado severamente

- Sentirse endurecido con la gente, levantarse fatigado por las mañanas y tener hijos tiene un $\mathbf{9 3 . 7 5 \%}$ de probabilidad de estar emocionalmente agotado todos los días

- Sentirse inquieto e inseguro, sentirse nervioso y sentirse desgastado por el trabajo indica que existe un $100 \%$ de certeza de padecer ansiedad severa

- Tener problemas digestivos, ser del sexo femenino, cansarse por trabajar con la gente, sentirse fatigado y agotado emocionalmente tiene un $96 \%$ de probabilidad de manifestar estrés laboral asistencial en su nivel más alto

- Tener ansiedad, sentirse agitado, cansarse por trabajar con la gente y estar fatigado tiene un $90 \%$ de probabilidad de padecer depresión mayor

\section{DISCUSIÓN}

Los estudios del estrés laboral asistencial involucran una gran cantidad de variables, diseños, áreas y profesiones, tanto en el ámbito de la salud como cualquier otro que este en contacto con la atención de personas, del cual se adquiere una variedad de información. Es relevante resaltar que, coincidiendo con Basset Machado, ${ }^{9}$ Arias Gallegos ${ }^{10}$ y Landa Ramírez, ${ }^{11}$ el sexo predominante son las mujeres, superando más de la mitad de la población, además de que la profesión de enfermería es estudiada en todas las investigaciones de referencia, siendo así una profesión que predomina en el área de la salud.

Las prevalencias entorno al estrés laboral asistencial fueron del $100 \%$ en la población, al igual que la ansiedad con el $100 \%$, aunque descritas por niveles fueron superiores los niveles bajos. Además, la depresión solo está presente en el 16.3\% de la población, al igual que Ovalles $\mathrm{Pérez}^{8}$ menciona porcentajes del $100 \%$ de estrés laboral y por encima del $50 \%$ en la ansiedad y depresión. Siendo así, se concuerda en que el ambiente laboral constituye un factor interviniente, el cual puede ser modificable para disminuir el estrés laboral asistencial, ya que los 
establecimientos y las permanencias de las condiciones laborales conllevan volver a un ciclo en el cual puede verse afectado el rendimiento del personal sanitario.

En cuanto a las tres dimensiones que conforman el diagnóstico para el estrés laboral, es necesario desglosarlas. Teniendo en la investigación niveles perniciosos bajos, como el agotamiento emocional y la despersonalización con cifras por debajo del $10 \%$ (7.0\% y 5.8\%, respectivamente), a diferencia de un $45.3 \%$ en un nivel bajo de realización personal presente en todas las profesiones del estudio a diferencia de odontología, cabe mencionar que se coincide con Basset Machado9 en su estudio en una unidad de psiquiatría, ya que refiere que el $3.3 \%$ presentó un nivel alto de agotamiento emocional, el $25 \%$ un nivel alto de despersonalización y el $76.7 \%$ un bajo nivel de realización personal. A pesar de que en el estudio de Basset Machado supera el 70\% y esta investigación solo el $40 \%$, ambos concuerdan en que es la dimensión más afectada, ya que, se sabe, es la dimensión que demuestra un bajo nivel de competencia y baja realización con éxito de las tareas laborales que se desempeñan, además de ver el trabajo de forma negativa al no conseguir objetivos propios en relación con el trabajo y la satisfacción personal. De esta forma, la realización personal en la población se ve afectada, teniendo un autoconcepto negativo, un distanciamiento de actividades familiares/sociales y una baja autoestima profesional.

En el hospital de psiquiatría se tiene la prestación de acudir a terapia psicológica como método de desintoxicación por el trabajo con los pacientes, no obstante, solo el $17.4 \%$ acude a recibir terapia de tipo psicológico, dejando a los demás expuestos a mantener o intensificar padecimientos de tipo psicológico por los diversos factores, ya sean desencadenantes o facilitadores. Madrigal Solano afirma que el tener alguna forma o tipo de trastorno suele afectar a los demás compañeros, al entorno familiar y hasta al mismo paciente por frecuentes actitudes negativas, sarcásticas, lo cual dificulta la dinámica social, y genera que el paciente pueda percibir mayor apatía de las personas, afectando su interacción y comunicación. ${ }^{5,6}$

Arias Gallegos ${ }^{10}$ refiere puntuaciones de $91.5 \%$ para el nivel moderado en la unificación del diagnóstico para el nivel del estrés laboral, mientras que los resultados de esta investigación denotan 36\% bajo, 60.5\% moderado y 3.5\% alto. Las prevalencias del nivel moderado son mayoría en ambos estudios, ya que existen puntuaciones desiguales y mayoritarias, sobre todo en el nivel de despersonalización (25\%) y de realización personal (45.3\%). Es por ello que se concuerda en que estas áreas son proclives a desencadenar una prevalencia del personal con falta de contacto con la realidad, ya que esta es una característica de la despersonalización, además de mostrar desinterés por el paciente y, por lo tanto, bajo involucramiento en el trabajo.

El presente estudio constituye un panorama hacia el objetivo general establecido, en el cual se pueden identificar padecimientos como la ansiedad, la depresión y el estrés laboral asistencial entorno al área de la salud mental. Es posible visualizar una relación estadísticamente significativa $(\mathrm{p}<0.05)$, a excepción de depresión y realización personal $(\mathrm{p}=0.19)$, al igual que Landa Ramírez. ${ }^{11}$ Al correlacionar dichas variables se obtuvo una relación baja y moderada, dando pie a la existencia de problemas laborales y emocionales, como se menciona anteriormente. Sin embargo, una relación baja refiere prevalencia baja por relación lineal, por lo cual es necesario considerar otras consecuencias 
en torno al estrés laboral asistencial. Como lo refiere Madrigal Solano, ${ }^{6}$ existen algunas otras, además de las psicológicas, lo cual se puede considerar para futuras investigaciones a fin de determinar padecimientos o situaciones con una fuerza alta, por lo que son áreas de oportunidad que se pueden trabajar con el personal sanitario para, al mismo tiempo, jerarquizar y priorizar las situaciones que se puedan tratar de resolver en primera instancia.

El poder describir cada uno de los ítems de los instrumentos empleados demuestra un área de oportunidad de manera independiente, ya que la puntuación total menciona un panorama en general, en el cual, describiéndolo y ordenándolo de manera descendente en prevalencia, se logran detectar situaciones específicas que pueden mejorarse y tratarse. Los de mayor prevalencia fueron: sentirse agotados $66.3 \%$, sentirse fatigados $54.3 \%$, con cansancio $52.3 \%$, desgastado $50 \%$, dureza en relación con la gente $50 \%$, sentirse acalorados $47.7 \%$, frustrados $43 \%$, insomnio inicial y medio $38.3 \%$, agitación $34.9 \%$, incapacidad de relajarse $30.3 \%$, entre otras. Cabe mencionar que la descripción de la dimensión de baja realización personal, al tratarse de preguntas con una afirmación positiva en torno a la profesión, conlleva interpretaciones de manera inversa, ya que esta fue la mayor oportunidad de mejora por ser prevalencias en sentido negativo en torno de una realización en el ámbito laboral. Esta manera de describir los ítems solo demuestra un sí (presente) o un no (ausente), pero el uso de los instrumentos y las puntuaciones totales definen el nivel de la presencia de alguna de ellas; es por eso que, de manera global, se obtuvieron valores bajos y, de manera específica, se demostraron valores altos. Así mismo, se demostró la fuerza de asociación existente en más de una de estas características.

Un dato más para analizar, que podría desencadenar un posible sesgo puesto que permitiría la presencia de niveles nocivos e, incluso, de mayor magnitud a lo obtenido en los resultados en torno a la salud mental del personal sanitario, es que el $46.5 \%$ de los participantes tenía algún conocimiento previo de los instrumentos aplicados y, siendo profesiones especializadas en el trato de padecimientos mentales, se puede llegar a deducir que existe una negación entorno a su propia salud mental, como lo menciona Moreno Jiménez et al. (2005). Como mecanismos de afrontamiento de negación, los profesionales suelen negar sus propias emociones y necesidades como forma de supervivencia, cerrándose emocionalmente y buscando mantener esa imagen social de equilibrio con la que comúnmente se les asocia. ${ }^{20}$ De ahí la importancia de incluir a todas las profesiones más allá de medicina y enfermería en trabajos de investigación, pues es vital para comprender la magnitud de la salud mental de los profesionales que componen la institución.

El análisis de los resultados genera nuevas interrogantes, las cuales pueden ser consideradas para investigaciones futuras, como el examinar a detalle y por separado factores como las consecuencias del estrés laboral asistencial, ya que en este caso se demostró que indagar en cada ítem de los instrumentos independientemente revela información que, de manera general, puede pasar desapercibida porque las correlaciones por instrumentos resultaron bajas y moderadas, aunque de manera global revelan fuerzas con porcentajes por encima del 90\%; como fue en el caso de las reglas de asociación. Cabe mencionar que siempre se tomó en cuenta la limitación de estudio en torno al tamaño de la muestra, ya que, al realizar una prueba predictora más robusta, como una 
regresión logística binaria, se obtuvieron significancias por encima del 0.05. A pesar de ello, la finalidad de conocer fuerzas de relación en torno a las variables de interés fue cubierta satisfactoriamente. Es por ello que se propone considerar el aumento y selección para el tamaño de la muestra, cuidar la metodología y obtener información actualizada de las instituciones, para que, a partir de eso, se desarrollen nuevas hipótesis y se continúe con la línea de investigación, como, por ejemplo, si el hecho de tener un segundo trabajo influye de manera significativa en el desarrollo del Síndrome de Burnout y, de igual forma, si aumentar la demanda en el programa que ofrece la misma institución ayudaría con el problema.

\section{CONCLUSIÓN}

Se determinó que en el hospital de psiquiatría si existen este tipo de padecimientos e, igualmente, se observó la presencia de relación entre ellos, con una fuerza baja y relación lineal directa en la ansiedad, la depresión y el estrés laboral asistencial. Por ende, se rechaza la hipótesis H1. Por ello, es necesario que se implemente un programa interno de atención hacia el personal sanitario enfocado en las necesidades detectadas, ya que el área de realización personal es el de mayor afección -como el sentirse cansado por trabajar todo el día con la gente, la incapacidad para relajarse, el sentirse acalorado, además de la menor incidencia de ansiedad y depresión con niveles altos-, así como poner a su disposición algún programa enfocado en crear técnicas efectivas de relajación en ellos mismos. El estrés laboral afecta a muchas áreas de la vida, las cuales deben ser atendidas de manera individual, como sugiere Maslach y Jackson (1981). ${ }^{21}$ Además, se deben considerar los procesos cognitivos de auto evaluación de los participantes, y el desarrollo de estrategias cognitivo-conductuales que les permitan eliminar o mitigar la fuente de estrés, evitar la experiencia de estrés, o neutralizar las secuencias negativas de esa experiencia para adaptarse a las circunstancias; o, a nivel grupal, potenciar la formación de las habilidades sociales y de apoyo social de los equipos de trabajo.

\section{Referencias}

1. Grau A, Suñer R, García MM. Desgaste profesional en el personal sanitario y su relación con los factores personales y ambientales. Gac Sanit [Internet]. 2005 [Consultado 1 Jun 2020]; 19(6):463-70. Disponible en: http://scielo.isciii.es/ scielo.php?script $=$ sci_arttext\&pid=S0213-91112005000600007\&lang=en

2. Roger MC. Burnout syndrome in nurses of Cuban cancer hospitals. Effectiveness of an intervention program. 2004-2009. Esc Nac Salud Pública [Internet]. 2009; Disponible en: http://www.iaea.org/inis/collection/ NCLCollectionStore/_Public/45/107/45107433.pdf

3. Avendaño C, Bustos P, Espinoza P, García F, Pierart T. Burnout y apoyo social en personal del servicio de psiquiatría de un hospital público. Cienc y Enferm [Internet]. 2009 [Consultado 1 Jun 2020]; 15(2):55-68. Disponible en: https://scielo.conicyt.cl/scielo.php? script=sci_arttext\&pid=S0717-95532009000200007\&lng=es\&nrm=iso\&tlng=es 
4. Olivares Faúndez V. Lauditorio: Dra. Chistina Maslach, Comprendiendo el Burnout. Ciencias Trab [Internet]. 2017 [Consultado 10 Jun 2020]; 19(58):59-63. Disponible en: www.cienciaytrabajo.cl

5. Bianchini Matamoros M. El Síndrome del Burnout en personal profesional de la salud. Med Leg Costa Rica [Internet]. 1997 [Consultado 1 Jun 2020]; 13-14(2-1-2):189-92. Disponible en: https://www.scielo.sa.cr/scielo.php? script $=$ sci_arttext\&pid $=$ S1409-00151997000200017

6. Madrigal Solano M. "BURNOUT" en Médicos: Aproximaciones teóricas y algunos diagnósticos direfenciales. Rev Med Leg Costa Rica [Internet]. 2006 [Consultado 1 Jun 2020]; 23(2):137-53. Disponible en: https://www.scielo.sa.cr/pdf/mlcr/ v23n2/3477.pdf

7. Valdes BC, Austria-Corrales F, Herrera Kiengelher L, Vázquez-García JC, Valero CZV, Salas-Hernández J. Estrategias activas de afrontamiento: Un factor protector ante el síndrome de burnout "o de desgaste profesional" en trabajadores de la salud. Rev del Inst Nac Enfermedades Respir [Internet]. 2010; 69(January):137-42. Disponible en: https://www.researchgate.net/ publication/287466514

8. Ovalles Pérez MA, Uribe Nobrega JC. Estrés laboral, ansiedad y depresión en residentes de Medicina Interna y Cirugía General de un hospital público de Aragua. Rev Mex Salud en el Trab [Internet]. 2014 [Consultado 16 Jul 2020]; 6(16):69-76. Disponible en: https://fenastac.org.mx/wp-content/ uploads/2015/02/REMESAT-EDICIÓN-16.pdf

9. Basset Machado I, Estévez Ramos R, Leal García ML, Guerrero Lara MG del $\mathrm{R}$, López Vázquez JT. Síndrome de Burnout en el personal de enfermería en una Unidad de Psiquiatría. J Petrol [Internet]. 2011 [Consultado 16 Jul 2020]; 10(1):252-66. Disponible en: https://www.medigraphic.com/pdfs/ enfneu/ene-2011/ene112f.pdf

10. Arias Gallegos WL, Muñoz del Carpio Toia A. Síndrome de burnout en personal de enfermería de Arequipa. Rev Cuba Salud Pública [Internet]. 2016; 42(4):55975. Disponible en: http://scielo.sld.cu/

11. Landa-Ramírez É, Rangel-Domínguez NE, Villavicencio-Carranza MA, WeingerzMehl S, Reyes-Saavedra MI, González-Álvarez VR et al. Clima organizacional y factores relacionados con el burnout en urgencias médicas: Un estudio correlacional. Psicol y Salud [Internet]. 2017; 27(2):245-54. Disponible en: http://psicologiaysalud.uv.mx/index.php/psicysalud/article/view/2525/4407

12. Sanz J. Recomendaciones para la utilización de la adaptación española del Inventario de Ansiedad de Beck (BAI) en la práctica clínica. Clínica y Salud [Internet]. 2014;25(1):39-48. Disponible en: https://journals.copmadrid.org/ clysa/art/cl2014a3

13. Banco de Instrumentos y Metodologías en Salud Mental. Ficha técnica del instrumento BAI [Internet]. CIBERSAM. 2015 [Consultado 2 Jun 2020]. p. 10. Disponible en: https://bi.cibersam.es/busqueda-de-instrumentos/ficha?Id=104

14. Banco de Instrumentos y Metodologías en Salud Mental. Ficha técnica del instrumento Hamilton [Internet]. CIBERSAM. 2015 [Consultado 27 May 2020]. p. 10. Disponible en: https://bi.cibersam.es/busqueda-de-instrumentos/ ficha? Id $=57$

15. Cañadas De La Fuente GA, Luis CS, Lozano LM, Vargas C, García I, De La Fuente EI. Evidencia de validez factorial del Maslach Burnout Inventory y estudio de los niveles de burnout en profesionales sanitarios. Rev Latinoam Psicol [Internet]. 2014 [Consultado 2 Jun 2020]; 46(1):44-52. Disponible en: http://zl.elsevier.es 
16. González Correales R, De la Gándara Martín J. ¿Cómo se detecta y mide el burnout? Rev Retos en la Salud Ment del Siglo XXI en Atención Primaria Soc Española Med Rural y Gen [Internet]. 1981 [Consultado 23 Jun 2020]; 16-9. Disponible en: http://horarioscentros.uned.es/archivos_publicos/ qdocente_planes/394118/ejdetectarbournout.pdf

17. Arturo J-G, Álvaro J. I, Anabel C-Á, Omar P-R. Síndrome de burnout en población mexicana: Una revisión sistemática. Salud Ment [Internet]. 2014 [Consultado 16 Jul 2020]; 37(febrero 2016):159-76. Disponible en: http://www.scielo.org.mx/ scielo.php?script=sci_arttext\&pid=S0185-33252014000200010

18. Jiménez-Blanco S, Frútos-Llanes R, Blanco-Montagut LE. Síndrome de Burnout: estudio de prevalencia y factores de riesgo en los profesionales de enfermería de atención primaria de Ávila. Rev Enfermería CyL [Internet]. 2011; 3(2):13-24. Disponible en: https://vpnuc.unican.es/index.php/revistaenfermeriacyl/article/ view/183/,DanaInfo=www.revistaenfermeriacyl.com +163

19. Ley General de Salud. Reglamento de la Ley General de Salud en materia de investigación para la salud [Internet]. Diario Oficial de la Federación. 2007 [Consultado 23 Jun 2020]. p. 1-30. Disponible en: http://www.salud.gob.mx/ unidades/cdi/nom/compi/rlgsmis.html

20. Moreno-Jiménez B, Rodríguez-Carvajal R. El burnout médico: la ansiedad y los procesos de afrontamiento como factores intervinientes. ANSIEDAD Y ESTRÉS [Internet]. 2005 [Consultado 25 Oct 2020]; Disponible en: https:// www.researchgate.net/publication/230596319

21. Apiquian Guitart A. El Síndrome del Burnout en las Empresas [Internet]. Universidad Anáhuac México Norte. 2007. Disponible en: https://www.ucm.es/ data/cont/media/www/pag-30022/sindrome burnout.pdf 